anti-predator behaviour. This is incorrect. Our experimental rationale relies instead on the assumption that any direct effects of parasitism of aphid escape behaviour are not significantly different between the two aphid biotypes. This does not require homogeneity at all other loci between the two populations. It is inconceivable, however, that aphids from the dry habitat are simply more susceptible to the direct effects of the parasite. Because of limits on space in our letter we were unable to substantiate the assumption that there were no differences in the direct effects of parasitism. In fact, we have found that aphids from the interior will refrain from suicidal behaviour when their reproductive potential exceeds zero (that is, when parasitized at a later age but assayed at the same age as before) $)^{3}$. Thus, these aphids clearly were not simply more susceptible to direct effects of parasitism than were aphids from the coastal region.

Second, Tomlinson, suggests that aphids from each region should be cross-infected by parasites from the other region to remove possible confounding effects of parasite origin. These experiments have been completed and they provide virtually identical results ${ }^{3}$. Thus, parasite source cannot explain the different behaviour of coastal and interior aphids.

Third, Tomlinson questions our use of the same on-ground survivorship values for both parasitized and unparasitized aphids. Although we have not measured these values for the former, it is highly probable that they are very similar to the latter, given the extreme temperatures found at ground level in the interior of British Columbia ${ }^{4}$. In fact, one might expect the more stressed parasitized individuals to be even more likely to succumb to environmental stress. Even if, for some remarkable reason, parasitized aphids were able to withstand heat stress to a greater degree than unparasitized individuals, it is unlikely that their survivorship under such circumstances would begin to approach that of aphids remaining on the plant, given the almost twofold difference between these options for unparasitized individuals. Similarly, observations indicate that behaviour following a back up or drop response is not different between parasitized and unparasitized aphids. Thus, we have reason to believe that mortality consequences of the different responses are significantly different between parasitized and unparasitized individuals.

Fourth, we agree that we have not demonstrated that the behaviour is under

\footnotetext{
1. McAllister, M. K. \& Roitberg, B. D. Nature 328, 797-799

(1987).

2. Tomlinson, I. Nature 330, 701 (1987)

3. McAllister, M. K. thesis, Simon Fraser Univ. (1987)

4. Roitberg, B. D. \& Myers, J. H. Can. Ent. 111, 515-519 (1979).

5. Latta, B. Nature 330, 701 (1987)

6. Roitberg, B. D., Myers, J. H. \& Frazer, B. D. J. anim. Ecol.
} 49, $111-122(1987)$. genetic control. What we have done, however, is to show that under identical environmental conditions, aphids from two regions respond differently to parasitism. Also, given our lack of knowledge of the genetics underlying these behaviours, it is not clear what the crossing experiments Tomlinson suggested would tell us.

Fifth, Tomlinson makes a series of essentially hand-waving gestures as regards evolutionary stable strategies (ESSs) and the apparent surprising subtlety of the behavioural change. We argue that our original proposition is parsimonious in that it does not require additional unfounded assumptions that the various behaviours have similar payoffs. In fact, the payoffs do differ and each behaviour appears to be used at different frequencies under different conditions, that is, they do not exist in a mixed ESS. With regard to the subtlety of the effect, we remind readers that though such modifications may appear slight in the laboratory, they may be much more pronounced in the field. Also, the results are statistically significant in the predicted direction.

Finally, Tomlinson's argument that asymmetries of selection between hosts and their parasites should favour parasites is unfounded. There are several conditions in which selection on the parasite may not be able to override concurrent selection for suicidal behaviour by hosts: (1) suicidal behaviour is triggered by a complex set of stimuli - this decreases the likelihood that variation in the parasite will be present for traits that could act to subvert this complex behaviour; and (2) the costs of maintaining a host-control mechanism is high relative to the payoff. For example, parasites with exceptionally high fecundity (for instance Aphidius ervi $\sim 567$ ) may find it exceedingly costly to provide each egg with sufficient neurotoxins to alter the behaviour of every host.

We now turn to Latta's ${ }^{5}$ suggestion that aphids from the coast should drop from plants as frequently as interior aphids because, once off the plant, they are as likely to infect non-kin as kin. If this were the case, we would concur with Latta. In fact, however, it is probably untrue that aphid colonies on 'nearby' plants are more likely to be non-kin, than kin, given the nature of wingless aphid dispersal ${ }^{6}$. Also given the nature of parasite mobility while searching for hosts, it is unlikely that aphid movement to another plant would provide that aphid with sufficient isolation to protect the colony from which it had moved. Isolation is a function of: (1) an aphid's position in space relative to the spatial and genetic structure of aphid populations; and (2) parasite search. We recently developed a mathematical model that explicitly considers the isolation factor and will report on it elsewhere.

Both Tomlinson and Latta question whether the original hypothesis of adap- tive suicide has been confirmed. We close by reminding scientists that experiments are abstractions that allow us to make inferences about real world events. Nowhere have we argued that we have, in fact, confirmed the host suicide hypothesis but rather, that under a set of well controlled experiments we failed to disprove it.

Murdoch K. MCAllister BERNARD D. ROITBERG

Behavioural Ecology Research Group,

Department of Biosciences,

Simon Fraser University,

Burnaby $B C$,

V5A 1S6, Canada

\section{A new parameter for sex education}

SIR-There is concern in West Germany over the falling birth rate. The accompanying graph ${ }^{1,2}$ might suggest a solution that every child knows makes sense.

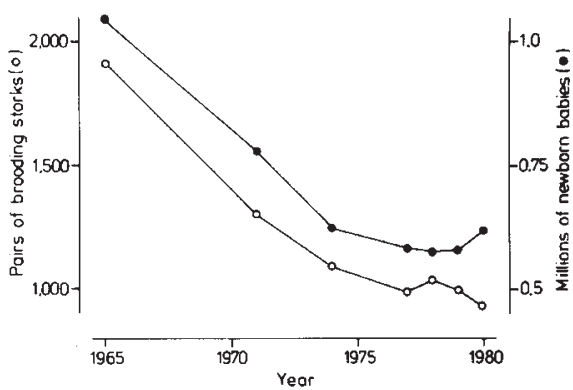

Helmut Sies

Institut für Physiologische Chemie 1, Universität Düsseldorf, Moorenstrasse 5, D-4000 Düsseldorf, FRG

1. Fachserie Gebiet und Bevölkerung (Statistisches Bundesamt, Kohlhammer, Stuttgart, 1984)

2. Bauer, S. \& Thielcke, G. Die Vogelwarte 31, 183-191 (1982).

\section{What are the masses of elementary particles?}

SIR-Numerology on the masses of the 'elementary particles' is usually refuted when the experimental results become more accurate, but I now deal with an example that has more than survived the ravages of time and which is to some extent related to the speculations of Eddington ${ }^{1}$.

Eddington believed that the fundamental constants could be simply expressed in terms of the integers $4,6,10$, $16,120,136,256$ and 137 which, apart from 137 , arise naturally when discussing tensors related to space-time. The prominence of 120 and 136 in Eddington's work was such that he called 136 the "basal multiplicity" and 120 the "number of dormant components in the extended energy tensor". These two numbers can also be described as the numbers of independent real and imaginary elements in a 16 by 16 Hermitian matrix, while 10 and 6 are the 